\title{
Haplotype-aware modeling of cis-regulatory effects highlights the gaps remaining in eQTL data
}

\section{Authors}

Nava Ehsan ${ }^{1}$, Bence M. Kotis ${ }^{1}$, Stephane E. Castel ${ }^{2,3}$, Eric J. Song ${ }^{1}$, Nicholas Mancuso ${ }^{4}$, Pejman Mohammadi ${ }^{1,5}$

\section{Abstract}

Expression Quantitative Trait Loci (eQTLs) are critical to understanding the mechanisms underlying disease-associated genomic loci. Nearly all protein-coding genes in the human genome have been associated with one or more eQTLs. Here we introduce a multi-variant generalization of allelic Fold Change (aFC), aFC-n, to enable accurate quantification of the cisregulatory effects in genes with multiple conditionally independent eQTLs. Applying aFC-n to 458,465 eQTLs in the Genotype-Tissue Expression (GTEx) project data, we demonstrate significant improvement in accuracy over the current tools for estimating the eQTL effect size and predicting genetically regulated gene expression. We characterize some of the empirical properties of the eQTL data and use this framework to assess the current state of eQTL data in terms of characterizing cis-regulatory landscape in individual genomes. Notably, we show that $77.4 \%$ of the genes with an allelic imbalance in a sample show $0.5 \log _{2}$ fold or more of residual imbalance after accounting for the eQTL data underlining the remaining gap in characterizing regulatory landscape in individual genomes. We further contrast this gap across tissue types, and ancestry backgrounds to identify its correlates and guide future studies. 
Genetic variation in the regulatory genome plays a major role in human phenotypic variability and disease susceptibility ${ }^{1}$. Large-scale expression quantitative trait loci (eQTL) mapping efforts in the past decade have identified thousands of common regulatory variants in the human genome that affect gene regulation ${ }^{2-7}$. This data is instrumental for understanding dosage-driven sources of phenotypic variation across individuals and interpreting the statistical signals from the genome-wide association studies (GWAS) ${ }^{8-12}$. For a given eQTL variant the regulatory effect size can be measured by allelic fold change (aFC), which is the fold difference between the expression of haplotypes carrying the reference and the alternative allele ${ }^{13}$. As a unified framework for modeling genetic effects in gene expression and ASE data, aFC is used in a wide range of applications ${ }^{3,4,11,14-19}$.

With the increasing sample size of eQTL transcriptome profiling studies, independent cis-eQTL mapping strategies have been developed to identify multiple eQTL signals for each gene in a population ${ }^{3,20-22}$. Notably, the Genotype-Tissue Expression (GTEx) consortium recently used a stepwise regression strategy to map conditionally independent cis-eQTL signals in 15,201 RNAsequencing samples of 838 post-mortem donors across 49 tissue sites ${ }^{3}$. This analysis demonstrated that virtually all protein-coding genes are affected by common genetic regulatory variants with a considerable level of allelic heterogeneity (Fig.1A). With larger eQTL studies already underway, it is expected that independent cis-eQTL signals will be mapped for an increasing number of genes ${ }^{7,23,24}$ (Supplementary Fig.1). However, there are currently no methods available for estimating the aFC effect sizes for independent eQTLs.

Here we introduce a multi-eQTL generalization of the aFC method, aFC-n, for estimating regulatory effect sizes from independent eQTL mapping studies. We benchmark the effect size estimates by aFC-n against those used in the GTEx v8 release ${ }^{3,13,14}$ and characterize their empirical properties and biological correlates. We assess the completeness of eQTL data in terms of characterizing cis-regulatory landscape in individual genomes and contrast it across 
tissue types, and ancestry backgrounds to identify its correlates and guide future studies. Finally, we provide tools and resources to estimate effect sizes and impute gene and haplotypespecific expression using conditional eQTL data.

\section{Results}

Multi-variant generalization of allelic effects. Under the aFC model, the expression associated with the reference $\left(e_{\mathrm{R}}\right)$ and the alternative $\left(e_{\mathrm{A}}\right)$ eQTL alleles in an individual are determined by a shared basal gene expression, $e_{\mathrm{B}}$, and allele-specific regulatory activities, $k_{\mathrm{R}}$, and $k_{\mathrm{A}}$ such that $e_{R}=e_{B} k_{R}$, and $e_{A}=e_{B} k_{A}$. The total gene expression in an individual is the sum of the two allele-specific expressions. The regulatory effect size, aFC, is $\delta_{A, R}=\frac{k_{A}}{k_{R}}$, which for a single eQTL, can be calculated using the previously published aFC quantification tool, hereafter referred to as $a F C-1^{13}$. Here, we introduce $a F C-n$ generalizing the model to a haplotype with $N$ independent eQTLs. The allele-specific expression in aFC-n is $e_{i_{1} \ldots i_{N}}=e_{R} \prod_{n=1}^{N} \delta_{i_{n}, R}^{(n)}$, where $i_{n}$ and $\delta_{i_{n}, R}^{(n)}$ are the present allele, and the associated aFC for the $n^{\text {th }}$ eQTL, respectively, and $e_{\mathrm{R}}$ is the expression of a haplotype carrying reference allele for all eQTLs. We infer the maximum likelihood parameters for this model under log-normal assumption to estimate aFC associated with all independent eQTLs affecting a gene using phased genotypes and gene expression counts (Methods). 


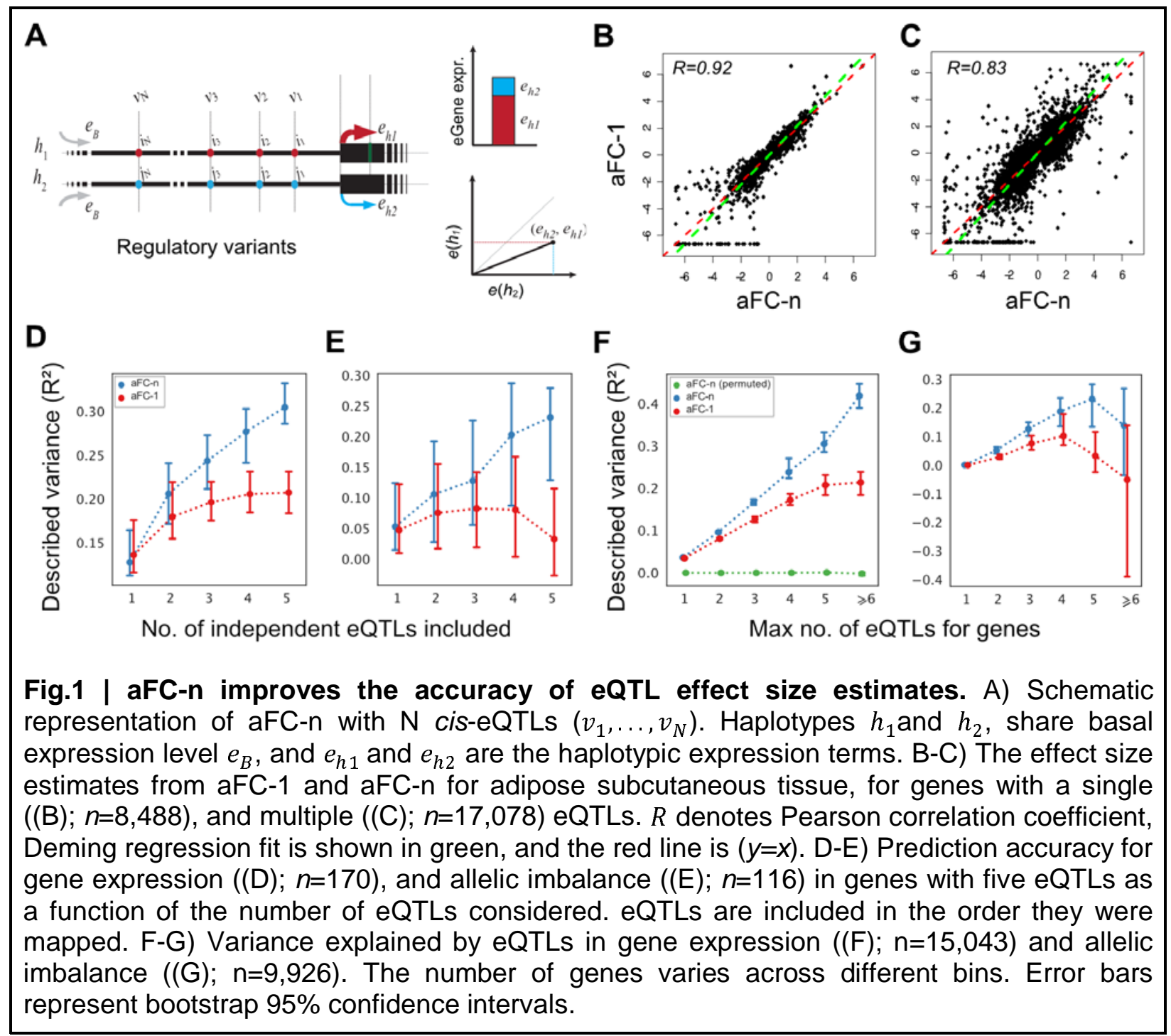

The aFC-n improves the accuracy of the cis-regulatory effect size estimates. To validate aFC-n, we used the empirical distribution of the adipose subcutaneous tissue in GTEx v8 eQTL data to simulate genetic regulatory effects in 15,167 genes with 1 to 14 eQTLs (Methods). In this simulation study, aFC-n consistently estimated the effect size accurately across all genes (Supplementary Fig.2). Applying aFC-n to GTEx project data v8, we estimated regulatory effect sizes for a total of 458,465 conditionally independent eQTLs from 49 tissues (Supplementary Fig.3). As expected, the effect size estimates from aFC-n were well correlated with the current effect size estimates from GTEx v8 eQTLs that were calculated using aFC-1. The correlation 
ranges from $89 \%-98 \%$ across tissues for genes with a single eQTL where the two methods are mathematically identical, and from $80 \%-92 \%$ for genes with multiple eQTLs (Fig.1B,C).

Next, we used adipose subcutaneous tissue data to compare the accuracy of the aFC estimates from the two methods in predicting gene expression and in predicting allele-specific expression across GTEx individuals (Methods). Since both methods are completely agnostic to allelespecific expression data, using allelic imbalance prediction accuracy allows us to evaluate the quality of the effect size estimates in an orthogonal way ${ }^{13}$. We compared the predicted gene expressions from each set of effect size estimates with the observed gene expression after logtransformation and PEER correction ${ }^{3}$. The predicted allelic imbalance was benchmarked against the observed logit-transformed haplotype-aggregated allelic expression generated by phASER ${ }^{14,25}$. Using genes that each have five conditionally independent eQTLs, we predicted gene and allelic expression five times each time including the effect size of one additional eQTL in the prediction. For the effect size estimates from aFC-1, we observed that including additional eQTLs leads to limited improvement in gene expression prediction accuracy and no increase in the prediction accuracy for allelic imbalance beyond what is achievable by accounting for the top eQTL genotype only. In contrast, the new effect size estimates by aFC-n delivered progressively better predictions as more eQTLs were included in the prediction of both gene and allelic expression (Fig.1D,E). Next, we used all genes with eQTLs to compare the overall prediction accuracy when all known eQTLs are considered for each gene. The accuracy gap between the predictions from aFC-n and aFC-1 was widened progressively in genes with more known eQTLs and overall the predictions were significantly more accurate for multi-eQTL genes (ranksum test p-value $7.36 \times 10^{-82}$ for gene expression, and $5.07 \times 10^{-8}$ for allelic imbalance) (Fig.1F,G). 
A

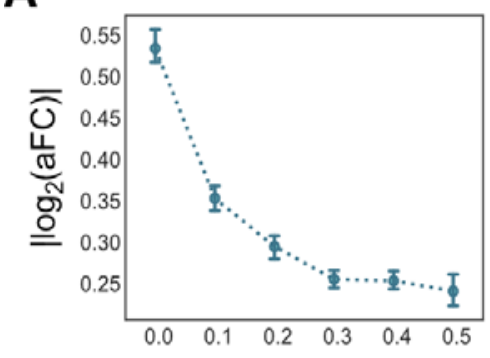

eQTL minor allele freq.(\%)

D

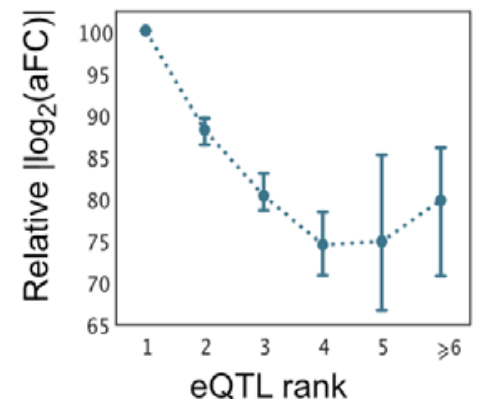

B

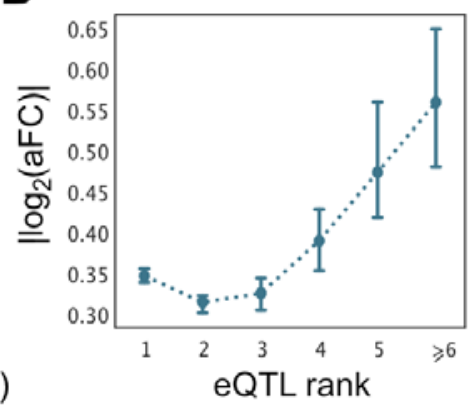

$E$

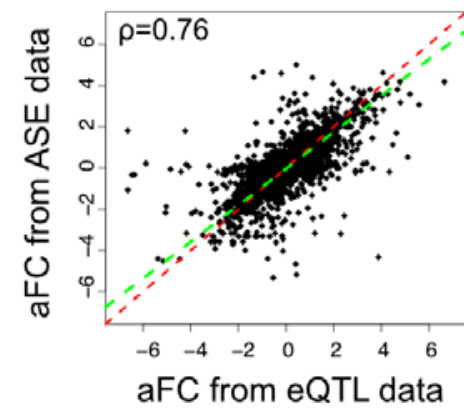

C

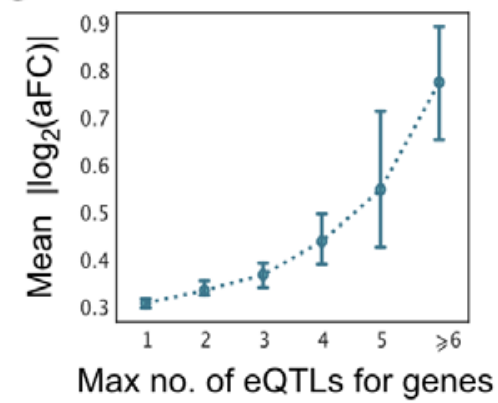

$\mathbf{F}$

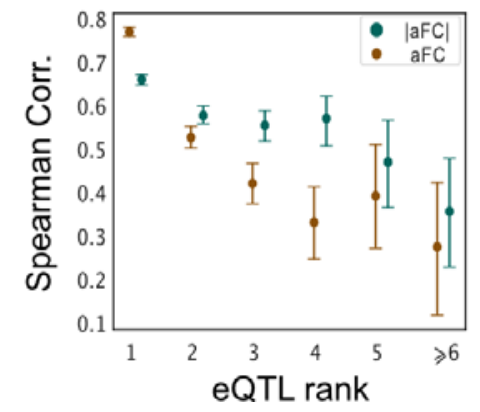

Fig.2 | Empirical properties of conditionally independent eQTL effect sizes. A-B) The absolute effect size as a function of eQTL minor allele frequency $(A)$ and the order at which it was identified for a gene (eQTL rank) (B) in adipose subcutaneous tissue. C) Average eQTL effect size as a function of the total number of eQTLs associated with a gene. D) The eQTL effect size relative to the average eQTL effect for a given gene as a function of the eQTL rank. E) The eQTL effect sizes as estimated by gene expression and population ASE data independently. The Deming regression fit is shown in green, and the red line is $(y=x)$. F) The correlation with the ASE data progressively decreased for secondary eQTLs. Error bars represent $95 \%$ bootstrap confidence intervals of the median.

Empirical properties of the estimated effect sizes. Next, we used aFC estimates from adipose subcutaneous tissue to characterize the regulatory effects of the independent eQTLs identified in GTEx project data. We found that $15.2 \%$ of the 25,675 independent eQTLs identified in adipose tissue altered the expression of a haplotype by more than two-fold (Supplementary Fig.4A). In addition, across all genes, secondary eQTLs (eQTLs were ranked with the order they were mapped in stepwise regression ${ }^{3}$ ) tended to have lower minor allele frequencies (Supplementary Fig.4B) and larger regulatory effects (Fig.2A-B). However, we found that the eQTLs in genes with many eQTLs tended to be stronger in general (Fig.2C). 
Accounting for this, we found that for a given gene the relative effect size of the eQTLs with respect to the average eQTL decreased with the order they were mapped (Fig.2D).

Next, we compared the cis-regulatory effects in eQTL and ASE data. We found that aFC effect sizes estimated from eQTL data were highly consistent with the median observed allelic imbalance among individuals that are heterozygous for the top eQTL, with sufficient $>10$ heterozygous individuals and minimum read coverage 8, (rank corr. 0.76 \pm 0.01 , Deming regression slope 0.9; Fig.2E). We further found that the concordance with the ASE data was decreased for secondary eQTLs (Fig.2F) partly due to the decreased minor allele frequency (Fig.2A; Supplementary Fig.4B), and partly due to the drop in haplotype phasing accuracy ${ }^{14,25}$.

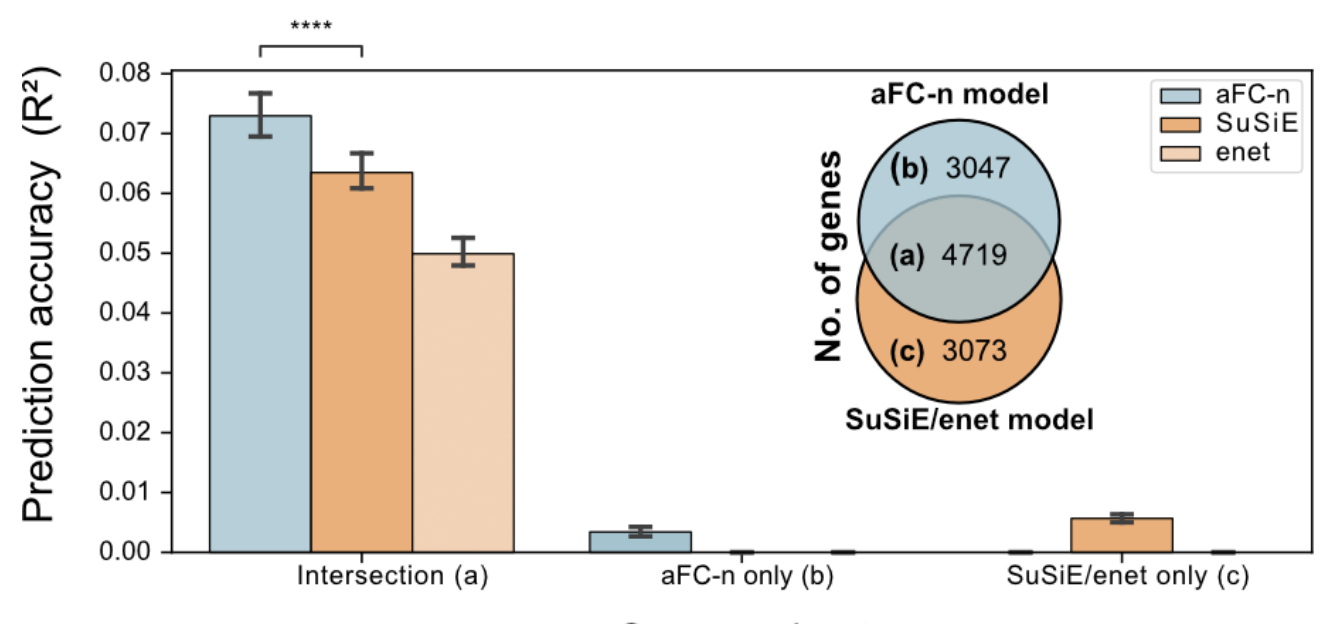

Genes subset

Fig.3 | The aFC-n improves predictive accuracy of genetically regulated gene expression. Comparing predicted gene expression from aFC-n, elastic net (enet) ${ }^{26}$ and SuSiE ${ }^{27}$ using out of sample data from adipose subcutaneous tissue. aFC-n outperforms SuSiE and elastic net for 4,719 genes shared by all models (Wilcoxon signed rank test $p$ value: $2.6 \times 10^{-32}$ ). For the genes not shared between models the performance is negligible for all models.

The aFC-n improves the prediction of genetically regulated gene expression. Next, we sought to demonstrate the application of aFC-n in predicting gene expression levels. Genetically driven gene expression has been widely used to identify transcriptome-mediated association signals in complex traits ${ }^{10,28}$. We used elastic net ${ }^{26}$ and $\mathrm{SuSiE}^{27}$, two powerful and robust 
methods used for predicting gene expression from genetic data to benchmark the accuracy of predicted gene expressions from eQTL effect sizes. We used GTEx v6p data from 316 adipose samples to build a predictive model using 11,146 conditionally independent eQTLs spanning over 7,766 genes (Methods) and evaluated the performance on 265 unseen samples exclusive to GTEx v8 release. For predicting expression using the aFC model we used independent eQTLs derived from GTEx v6p data (mean 1.4 eQTLs per gene), and for the other two models, we used all genetic variants in the $1 \mathrm{Mb}$ window around each gene meeting our $\mathrm{QC}$ criteria (Methods) restricting the comparison to genes with cis-heritability $p$-value $<0.01$ present in eQTL data $(n=4,719)$. We found that the prediction performance of the eQTL genotypes in the aFC model was higher than the two state-of-the-art methods in unseen samples (Fig.3). Consistent with previous reports ${ }^{29-31}$, we observed a notable reduction in prediction accuracy among African American ancestry individuals in all three models (Supplementary Fig.5A). To alleviate this issue, we devised a hierarchical extension of the aFC-n model to allow ancestryspecific aFC estimates when supported by data (Methods). We found that the ancestry-specific signals identified by this model were generally false positives driven by low sample sizes and therefore failing to diminish the performance gap between different ancestry groups (Supplementary Fig.5B). 


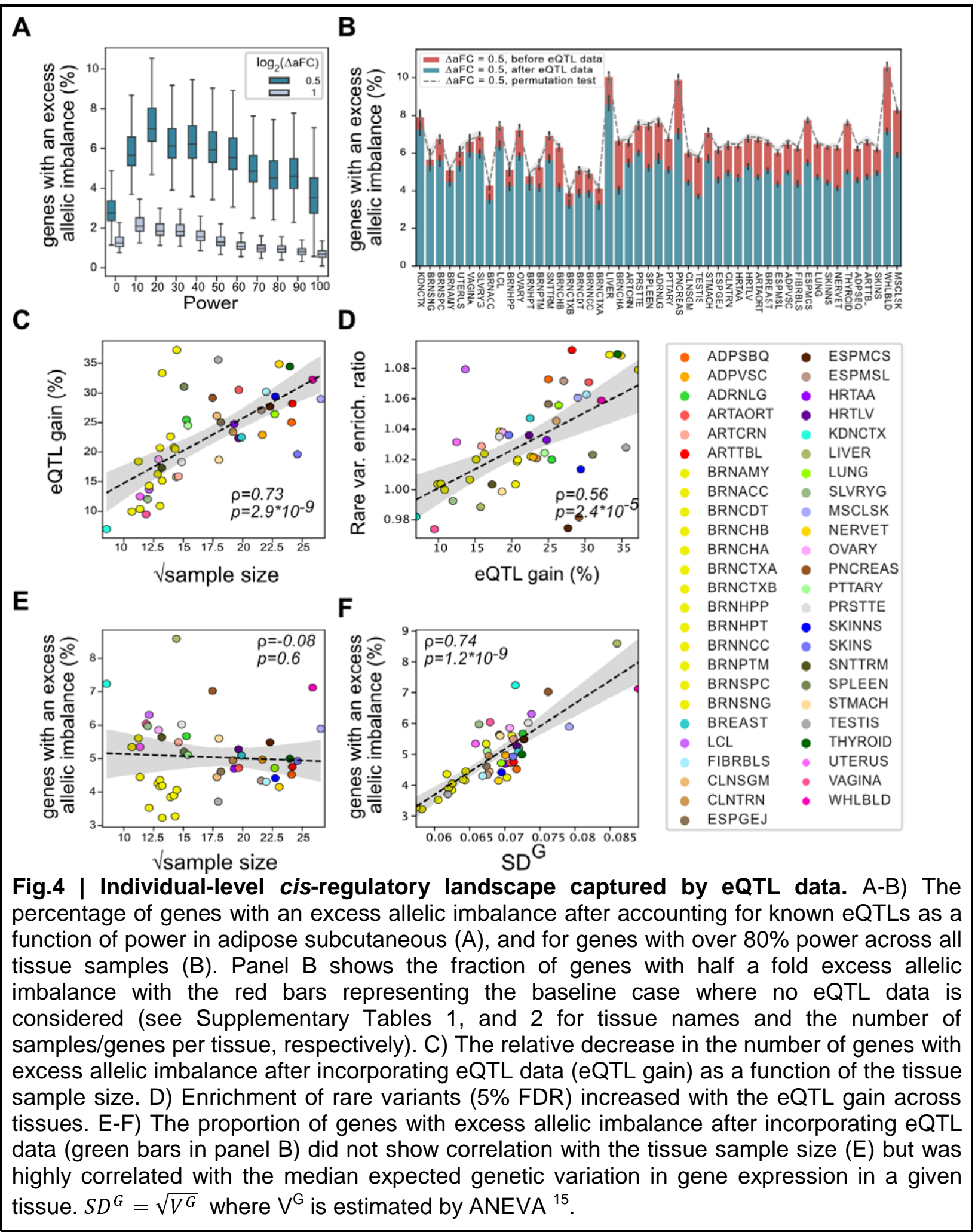




\section{Majority of the observed allelic imbalance in individual samples is not described by the}

current eQTL data. Next, we sought to determine the fraction of genes where the cis-regulatory landscape is adequately characterized at an individual level by their genotype at known eQTLs. We used the observed allelic imbalance in a gene as the ground truth under the rationale that the ASE data is the net regulatory effect of all heterozygous cis-acting variants affecting a gene - including those that are not known eQTLs. Furthermore, allelic imbalance is almost entirely driven by genetic factors, with heritability estimates above $85 \%{ }^{32}$. Specifically, for each gene in an individual, we checked if the allelic imbalance is consistent with predictions from the eQTL data and identified cases where we observed excess imbalance beyond 0.5 and 1 aFC (Methods). We performed power analysis to account for the confounding effect of limited statistical power in detecting an excess allelic imbalance in low expressed genes (Supplementary Fig.6). Looking at the adipose subcutaneous tissue samples we found that the number of genes with excess allelic imbalance initially increased but then it dropped progressively with increased statistical power at the right end of the axis (Fig.4A). The unexpected drop in spite of the high statistical power to detect allelic imbalance in these genes is due to a general tendency in highly expressed genes to be intolerant of genetic variation (Supplementary Fig.6D-E) ${ }^{13,15,33}$. We limited our analysis to protein-coding genes expressed $>1$ TPM and $>80 \%$ statistical power to identify 0.5 fold resolution in $\log _{2}$ aFC scale. We found that on average between 3.2\% (Brain - Frontal Cortex (BA9)) and 8.6\% (Liver) of the considered genes across different tissues in an individual showed excess allelic imbalance beyond what is expected from the genotypes at known eQTL SNPs. This constituted on average a $22.6 \%$ decrease from a baseline scenario where no eQTL knowledge was available and both haplotypes were expected to be expressed equally in all genes (Fig.4B). This decrease represents the gene regulatory knowledge gained by the GTEx consortium eQTL analysis. As expected, the gain was highly correlated to the sample size of the tissues as more cis-regulatory variants were identified (Fig.4C; Supplementary Fig.7). The enrichment of rare variants among 
genes with excess allelic imbalance (5\% FDR) also increased with the gain across tissues, confirming that with increased statistical power the eQTL model better captures the common variant regulatory effects and the excess allelic effects by rare variants (Fig.4D). However, we found that in contrast to the relative gain, the absolute fraction of genes with an excess allelic imbalance in each tissue was not correlated with the sample size used in the eQTL analysis (Fig.4E) but instead was correlated with the median amount of heritable variation in gene expression $\left(V^{G}\right)$ in each tissue as estimated by the analysis of expression variation (ANEVA) ${ }^{15}$ (Fig.4F).
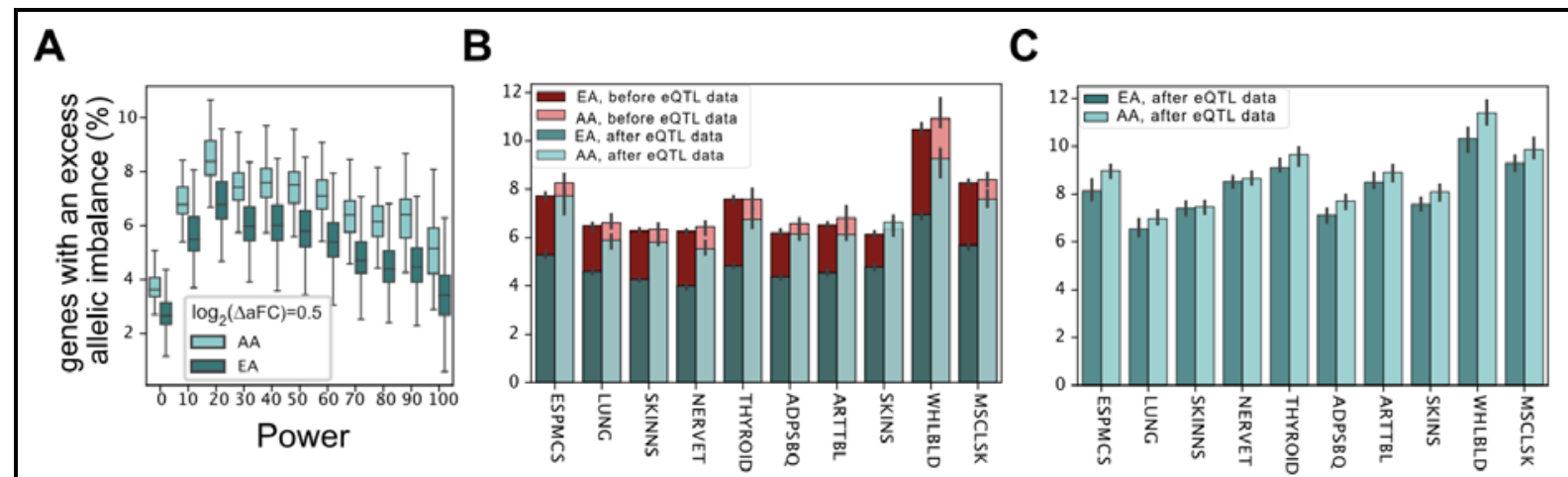

Fig.5 | Self-reported ancestry, and the accuracy gap in capturing individual-level cisregulatory landscape by $\mathbf{E Q T L}$ data. The percentage of genes with an excess allelic imbalance by self-reported ancestry after accounting for known eQTLs as a function of power in adipose subcutaneous (A), and for genes with over $80 \%$ power across ten GTEx tissues with largest sample sizes (B-C). $A A$ and EA denote African American, and European American individuals, respectively. The eQTL data used in panels $A$ and $B$ is from the official GTEx release, while for panel $C$ the eQTLs were mapped using a subset of the samples with equal number of $\mathrm{AA}$ and EA individuals. Error bars represent $95 \%$ bootstrap confidence intervals of the median (see Supplementary Table 3 for number of analyzed samples/genes per tissue/population).

\section{Matching sample counts alone will not close the accuracy gap in African ancestry}

individuals. GTEx data includes mostly individuals of European descent ${ }^{3}$. To assess how well the cis-regulatory landscape of the genes is captured across different ancestry backgrounds, we further stratified the analysis by self-reported ancestry for each GTEx donor. Looking at the adipose subcutaneous tissue samples, we found that the percentage of genes with excess allelic imbalance was significantly higher among African Americans suggesting that the eQTL 
data does not capture the cis-regulatory landscape in African ancestry individuals as accurately (ranksum test $p$-value $=2.3 \times 10^{-25}$ for genes with $80 \%$ power; Fig.5A). Looking at the ten most sampled tissues, we observed that the gain due to eQTL data is systematically lower in the African ancestry individuals in line with the lower sample sizes (Fig.5B). To exclude the effect of sample size, we repeated the eQTL mapping using the same number of European and African ancestry individuals for each tissue. We found that the number of genes with excess allelic imbalance was still higher in the African ancestry individuals (Wilcoxon signed-rank test, p-value $=0.002$; Fig.5C). While the number of samples used in the eQTL analysis was identical for both ancestry groups the remaining gap is likely due to a higher level of regulatory variation and/or reference bias in ASE data in the African American population (Fig.5C; Supplementary Fig.8) highlighting additional obstacles that impede analysis of non-European ancestry genomes.

\section{Discussion}

With the increasing size of the eQTL studies, many genes are associated with multiple eQTL variants. Here we introduced a new multi-variant method, aFC-n, to accurately estimate the cisregulatory effect size for independent eQTLs associated with a gene. Applying aFC-n to GTEx v8 project data we showed that the resulting eQTL effect sizes are significantly more accurate than the currently available estimates in predicting the cis-genetic effect on expression, and ASE data. Using these effect sizes to predict allelic imbalance, we showed that the current eQTL data is highly consistent with the observed allelic imbalance at the population level.

The extent of regulatory variation represented by eQTL data has been previously explored implicitly by heritability analysis ${ }^{32}$, and by quantifying the diminishing number of identified eQTLs at a certain sample size ${ }^{3,4}$. A comprehensive catalog of the eQTLs is critical for identifying dosage-driven phenotypic variation and GWAS interpretation ${ }^{8,9,11}$. Here we employed the aFC framework to explicitly assess the eQTL data in representing individual-level regulatory landscape using ASE data. We found that the current eQTL data from GTEx 
adequately represented the net cis-regulatory effects on the majority of the gene haplotypes across tissues. However, this is mainly due to the fact that most genes in a given sample do not show significant allelic imbalance. Controlling for the statistical power and at half a $\log _{2}$ fold resolution, we found that just over a fifth of the genes with allelic imbalance in a sample is accounted by the current eQTL data; indicating that a majority of cis-regulatory genetic variants, likely with low minor allele frequencies, are yet to be mapped. As expected, and in line with the increased power in eQTL mapping, the tissue types with larger sample sizes in GTEx data tended to show a higher gain in describing genes with allelic imbalance. However, the amount of residual regulatory variation across samples of a given tissue type was not correlated with the sample size, instead it was strongly correlated with the total amount of genetic regulatory variation in a given tissue as estimated by ANEVA. Considering that tissue samples are from the same individuals, it is implausible that this observation would be a technical artifact driven by reference bias in ASE data. We postulate that the adequate sample size for an eQTL study is not a fixed number and ultimately depends on the specific biological context being studied.

A similar analysis across European and African American individuals defined by self-reported ancestry demonstrated that the GTEx eQTL data offers lower gains in describing allelic imbalance in African American individuals and is overall less informative. This general issue is well recognized in the field and is in line with the fact that most individuals included in the GTEx project are of European ancestry ${ }^{3,34,35}$. However, we found that the performance gap between European and African Americans decreased but did not entirely disappear even when the sample sizes were matched. This observation was consistent with the higher amount of variation in ASE data as measured by ANEVA and in line with the extensive genomic diversity in Africa. However, unlike the cross-tissue analysis, we cannot rule out a contribution of technical artifacts due to reference bias. Nevertheless, the de facto performance gap, which goes beyond the sample size effects, suggest that reaching the same predictive accuracy in analyzing African American genomes will likely entail not only improvement in methods and references but also 
the inclusion of a relatively higher number of samples to enable characterization of an inherently more diverse and admixed population.

Finally, while haplotype-aware eQTL mapping methods have been proposed ${ }^{36-38}$, genome phasing quality remains a bottleneck in capturing the regulatory landscape in human haplotypes. We expect long-range phasing of the genomes beyond what is currently achievable using short-read sequencing ${ }^{25}$ to be a valuable addition to the reference transcriptomic cohorts such as GTEx, and TOPMed ${ }^{23}$. Transcriptome-wide association studies utilize genetically predicted gene expression to identify phenotypic variations that are likely mediated through genetically driven gene dosage. Our method is distinct from the current approaches in that it uses a mechanistic method to predict gene expression in a haplotype-specific fashion using a relatively small set of known eQTL variants. We showed that this model of genetic variation in cis-regulation yielded higher predictive accuracy than the state-of-the-art gene expression prediction methods. However, unlike regression-based methods, our method cannot be used to analyze associations based on summary statistics.

\section{Methods}

\section{Haplotypic aFC estimation}

The expression associated with eQTL alleles on each haplotype is described with a shared basal gene expression, $e_{B}$ and allele-specific factors $k_{R}$, and $k_{A}$. The regulatory effect size, aFC, is defined as $\delta_{A, R}=\frac{k_{A}}{k_{R}}$. Considering the case of $\mathrm{N}$ eQTLs acting on the same gene independently and defining $s_{A, R}=\log _{2} \delta_{A, R}$, the expression of the haplotype carrying $\mathrm{N}$ variants is:

$$
\log _{2} e_{i_{1} \ldots i_{N}}=\log _{2} e_{R}+\sum_{n=1}^{N} s_{i_{n}, R}^{(n)}
$$


where $s_{i_{n}, R}^{(n)}$ is the log aFC associated with the allele $i_{n}$ of the $n^{\text {th }}$ eQTL, and $e_{\mathrm{R}}$ is the expression of a haplotype carrying reference allele. This generalized aFC model is used in a variance stabilized nonlinear regression to estimate aFCs associated with all independent eQTL variants affecting a given gene, simultaneously. Assuming a multiplicative noise model ${ }^{13}$, the haplotypeaware aFC could be estimated as the least-squares solution to the following nonlinear equation:

$$
\log _{2} e_{<i_{1} \ldots i_{N}>,<j_{1} \ldots j_{N}>}=\log _{2}\left(2^{s . h 1}+2^{s . h 2}\right)+\log _{2} e_{R}+\varepsilon
$$

where $\left.e_{<i_{1} \ldots i_{N}>,<j_{1} \ldots j_{N}}\right\rangle$ is the total gene expression which is the sum of the two haplotypic counts, and $h_{1}$ and $h_{2}$ are binary indicator vectors representing the phased genotype of each allele, $s$ is the vector denoting the log-transformed effect sizes of eQTLs and $\varepsilon$ is a multiplicative noise.

To estimate the model parameters, we used gene expression read counts. We normalized the counts for library size, added 1 pseudo-count and log-transformed to stabilize the variance for least-square fitting. The expressions were corrected for significant linear effects of identified confounding factors using PEER ${ }^{39}$, top 5 genotype-based principal components, sequencing platform (Illumina HiSeq 2000 or HiSeq X), sequencing protocol (PCR-based or PCR-free) and sex. The correction was done in two steps: first, we regressed the expression vector of each gene against covariates and selected those with nominally significant coefficients $(p<0.01)$. Then we regressed the expression vector on selected covariates and set the residuals as the corrected expression vector which was used for effect size calculation ${ }^{13}$.

The log aFCs for eQTLs were calculated using non-linear least-squares regression and were constrained to $\pm \log _{2}(100)$ to avoid outliers. The initialization step is a linear fit where the haplotype vector was used as an independent variable and the adjusted expression was the dependent variable. The coefficients were used as the initial values of the vector $s$ in the nonlinear optimization function. This makes the LM algorithm converge closer to the real value. We used the Python non-linear least-squares minimization and curve fitting (LMFIT) library. 
Confidence intervals were calculated to infer $95 \%$ confidence intervals for the aFC estimates.

In GTEx v8 data, the range of those eQTLs whose 95\% confidence interval of aFC estimates overlapped zero varied from $10.2 \%$ (kidney-cortex) to $32.8 \%$ (cells-cultured-fibroblasts) across tissues if PEER correction was not included, and the range narrowed between $2.07 \%$ (BrainSubstantia-nigra) and $5.8 \%$ (Testis) when correcting for confounding variation by PEER factor on aFC estimates.

\section{Simulation scheme}

We applied our method on simulated data to evaluate how our haplotype-aware method for calculating aFC performs under expression noise level. In this designed simulation, we used the 15,167 genes in adipose subcutaneous with 1 to 14 eQTLs. The simulated measurements of aFCs came from a normal distribution norm[0, $\sigma=1]$. Poisson and binomial noise are added to expression and haplotype counts, respectively (Supplementary Fig.2).

\section{Allele-specific and gene expression prediction}

The estimated effect sizes were used to predict the cis-genetic effects on gene expression and allelic imbalance in individuals with phased genotype data. The prediction accuracy was measured by the square of Pearson correlation, $R^{2}$, between predicted and observed gene expression. The evaluation is assessed against gene expression after log-transformation and PEER correction ${ }^{3}$ and haplotype-aggregated allelic expression generated by phASER ${ }^{14,25}$. To smooth the haplotype counts, a pseudo-count of 0.5 was added to each observed haplotype expression and the minimum total coverage to be included in the calculations was set to 100 . This makes the data well powered to detect allelic imbalance (Supplementary Fig.6A; Supplementary Fig.9A). The log-transformed estimated gene expression $\left(e_{\text {total }}\right)$ and Allelic Imbalance $(A I)$ for an individual were derived from equation (Eq. 3), 


$$
\begin{gathered}
e_{\text {total }}=\log _{2}\left(2^{s . h 1}+2^{s . h 2}\right) \\
A I=s . h 1-s . h 2
\end{gathered}
$$

Where, $h_{1}$ and $h_{2}$ are binary indicator vectors representing the genotype of each allele of the individual and $s$ is the vector denoting the log-transformed effect sizes of eQTLs of interest and $s . h_{i}$ is the sum of effect sizes for alternative alleles.

To compare the accuracy of cis-regulatory variation described by the aFC estimates derived by the two methods (aFC-n and aFC-1), we used the effect sizes of 25,232 and 17,147 eQTL variants in adipose subcutaneous tissue samples from 581 individuals for gene expression and allelic expression, respectively. Due to the constraint on minimum coverage for ASE analysis, the number of individuals taken into account differs for each gene (Supplementary Fig.9).

First, the comparison was done based on the number of eQTLs included for each gene. By using the effect sizes derived from aFC-n model (Eq. 2), the median $R^{2}$ increased as more variants were taken into account for both gene expression and allelic imbalance (Fig.1C,D).

Both models performed similarly for genes with one eQTL but the portion of total expression variation that could be explained by the known eQTLs for gene expression and allelic imbalance showed an increasing pattern for the median $R^{2}$, when genes have more regulatory variants (Fig.1E,F). To examine over-fitting, a permutation test was applied to the data. This was done by shuffling the gene expression data over the individuals and training the model based on the shuffled data. The output showed that although the decreasing pattern was held, we did not observe similar performance from the shuffled data (Fig.1E).

The ASE prediction was compared with the read counts with WASP mapping strategy ${ }^{36}$ to reduce the mapping bias that is sometimes present in ASE analysis. On average, WASP correction improved prediction about 7 percent for 9,503 genes at a minimum coverage of 100 reads in adipose subcutaneous tissue. 


\section{Applying eQTL-based prediction models}

To compare aFC-n with eQTL-based prediction models, we fitted eQTL data for 316 individuals with measured gene expression in adipose subcutaneous tissue in the GTEx v6 using an elastic net and the Sum-of-Single-Effects model (i.e. SuSiE). For each gene, we focused on local genetic variation flanking $0.5 \mathrm{Mb}$ up/downstream of the gene body. We kept only bi-allelic SNPs that exhibited minor allele frequency 0.05 and HWE p-value $>1$ e-5 captured. We then fit elastic net and SuSiE models to the log-transformed gene expression data, adjusting for the top 5 genotyping PCs, sequencing protocol (PCR-based or -free), sequencing platform (Illumina HiSeq 2000 or HiSeq X), sex, and age. To evaluate the performance of these eQTL-based approaches, we then computed the out-of-sample $R^{2}$ using 265 newly added individuals in GTEx v8.

\section{cis-eQTL mapping}

To perform independent cis-eQTL mapping based on GTEx v6p samples, gene expression values from tissue samples were normalized and limited to autosomal genes with more than 5 reads in at least 10 individuals. The cis-eQTL mapping is performed using tensorQTL ${ }^{22}$, based on the stepwise regression approach described in ${ }^{3}$, using WGS-based genotypes. Restricting the minor allele frequency threshold to 0.01 , we obtained 11,146 independent cis-eQTLs associated with 7,766 genes for adipose subcutaneous tissue.

\section{Power analysis for ASE prediction}

We performed power analysis to estimate the fraction of the cases that the current eQTL data fully described ASE signal. Statistical power facilitates the interpretation of the results, where the low read counts or other features make it less likely to observe significant differences between the observed and predicted values. Factors that affect the power of the statistical test 
are the amount of total count, reference ratio, and the minimum fold change between the predicted and observed value.

For this purpose, we simulated the hypothesis $\left(H_{1}\right)$ in which the $\log (\mathrm{aFC})$ of data is systematically off from the null hypothesis $\left(H_{0}\right)$ by specific fold change $(f c)$ (Eq.4), assuming $H_{0}$ data is binomial distributed with null reference ratio $r_{0}$.

$$
\log (a F C)_{H 1}=\log (a F C)_{H 0} \pm f C
$$

The $\log (\mathrm{aFC})$ is defined as the logit function of the reference ratio $\left(r_{i}\right)$ (Eq.5).

$$
\log (a F C)_{H i}=\log \left(\frac{r_{i}}{1-r_{i}}\right)
$$

One thousand binomial samples were produced from the hypothesis $H_{1}$ (500 samples from $\log (a F C)_{H 1}=\log (a F C)_{H 0}+f c$, and 500 samples from $\left.\log (a F C)_{H 1}=\log (a F C)_{H 0}-f c\right)$ for different levels of read coverage and reference ratios. The significant difference between the generated samples of $H_{1}$ and the null hypothesis $H_{0}$ was determined by the binomial test (nominal $p$-value $<0.01$ ). Power estimation based on simulation for different amount of read counts and reference ratios considering specific fold changes $(\triangle a F C) 0.5$ and 1 were calculated (Supplementary Fig.6A). Here is an example to give an idea of how the fold resolution affects the reference ratios. Assuming the null hypothesis $\log _{2}(\mathrm{aFC})$ to be zero, the $r_{0}$ is 0.5 , the fold resolution of 0.5 and 1 corresponds to reference ratios of about 0.59 (or 0.41 ) and 0.67 (or 0.33), respectively (Supplementary Fig.6B).

Applying power statistics analysis on the GTEx v8 haplotype-aggregated ASE data, for each individual, the protein-coding genes with TPM $>1$ available at different levels of power (for 0.5 and 1 fold resolution) were considered for downstream analysis (Supplementary Fig.6C). The significance of $\triangle a F C$ (difference of aFCs between the predicted and observed values), was determined by the binomial test at a $1 \%$ p-value threshold with the cut-off of 0.5 (for 0.5 fold resolution) or 1 (for 1 fold resolution) for $\triangle a F C$ to avoid false positives in high expressed genes. 


\section{Calculating ancestry-specific aFC estimation}

We generalized the aFC-n model to calculate ancestry-specific aFC for European-American and African-American sub-populations using (Eq.6),

$$
\log _{2} e_{<i_{1} \ldots i_{N}>,<j_{1} \ldots j_{N}>}=\log _{2}\left(2^{\left(s_{E}(1-I)+s_{A} I\right) \cdot h 1}+2^{\left(s_{E}(1-I)+s_{A} I\right) \cdot h 2}\right)+\log _{2} e_{R}+\varepsilon
$$

where $h_{i}$ is a binary vector representing the phased genotype of each allele and $I$ is an indicator of ancestry background for each individual with a fixed value ( 1 for African-Americans and 0 otherwise). The estimates $s_{E}$ and $s_{A}$ represent the aFC for European and African populations, respectively. The ancestry-specific aFC was selected for cases with non-overlapping confidence intervals and for the rest of the cases we used effect size estimates derived from the standard aFC-n model.

\section{$V^{G}$ estimation}

$V^{G}$ estimates were calculated over GTEx v8 samples by the analysis of expression variation (ANEVA) ${ }^{15}$. We analyzed genes with at least 30 reads in at least 6 donors and at least 5,000 reads in all individuals in a target tissue. The median of $S^{G}\left(\sqrt{V^{G}}\right)$ was calculated for tested genes for each sample and the median of the resulting values was calculated across tissues (Fig.4F).

\section{Data availability}

The datasets analyzed during the current study are available to authorized users via dbGaP under accession phs000424 and on the GTEx portal (http://gtexportal.org/).

The aFC estimates for all independent eQTLs in GTEx v8 data are available on (https://github.com/PejLab/aFCs). 


\section{Code availability}

Software for calculating aFC from independent eQTL data is available online on (https://github.com/PejLab/aFC-n).

Software for calculating predicted ASE and gene expression using allelic fold change is available online on (https://github.com/PejLab/gene expr pred).

\section{Acknowledgments}

We thank the GTEx donors for their contributions to science, the GTEx Laboratory, Data Analysis, and Coordinating Center (LDACC), and the GTEx analysis working group (AWG) for their work in generating the resource.

\section{Author information}

${ }^{1}$ Department of Integrative Structural and Computational Biology, The Scripps Research Institute, La Jolla, CA, USA

${ }^{2}$ Department of Systems Biology, Columbia University, New York, NY, USA

${ }^{3}$ New York Genome Center, New York, NY, USA

${ }^{4}$ Center for Genetic Epidemiology, Department of Population and Public Health Sciences, Keck School of Medicine, USC, CA, USA

${ }^{5}$ Scripps Translational Science Institute, The Scripps Research Institute, La Jolla, CA, USA.

\section{Authors' Contributions}

N.E and P.M conceived the work and wrote the manuscript. B.M.K implemented the aFC pipeline. E.J.S calculated the genetic variation in gene expression estimates. N.M performed the SuSiE and elastic net analysis. S.E.C generated the haplotype-aggregated allelic expression data. N.E performed all other analyses. All the authors provided critical feedback on the 
manuscript.

\section{Ethics declarations}

Competing Interests

S.E.C. is a co-founder, Chief Technology Officer, and stock owner at Variant Bio.

\section{Funding}

N.E, N.M and P.M were supported by NIGMS award R01GM140287. N.E and P.M were

supported by a collaborative research agreement with Takeda California, Inc. S.E.C. was supported by NHGRI grant 1K99HG009916-01. 


\section{References}

1. Albert, F. W. \& Kruglyak, L. The role of regulatory variation in complex traits and disease. Nat. Rev. Genet. 16, 197-212 (2015).

2. Battle, A. et al. Characterizing the genetic basis of transcriptome diversity through RNAsequencing of 922 individuals. Genome Res. 24, 14-24 (2014).

3. GTEx Consortium. The GTEx Consortium atlas of genetic regulatory effects across human tissues. Science 369, 1318-1330 (2020).

4. GTEx Consortium et al. Genetic effects on gene expression across human tissues. Nature 550, 204-213 (2017).

5. Kerimov, N. et al. A compendium of uniformly processed human gene expression and splicing quantitative trait loci. Nat. Genet. 53, 1290-1299 (2021).

6. Lappalainen, T. et al. Transcriptome and genome sequencing uncovers functional variation in humans. Nature 501, 506-511 (2013).

7. Võsa, U. et al. Unraveling the polygenic architecture of complex traits using blood eQTL meta-analysis. BioRxiv (2018) doi:10.1101/447367.

8. Barbeira, A. N. et al. Exploring the phenotypic consequences of tissue specific gene expression variation inferred from GWAS summary statistics. Nat. Commun. 9, 1825 (2018).

9. Yao, D. W., O’Connor, L. J., Price, A. L. \& Gusev, A. Quantifying genetic effects on disease mediated by assayed gene expression levels. Nat. Genet. 52, 626-633 (2020).

10. Gamazon, E. R. et al. A gene-based association method for mapping traits using reference transcriptome data. Nat. Genet. 47, 1091-1098 (2015).

11. Castel, S. E. et al. Modified penetrance of coding variants by cis-regulatory variation contributes to disease risk. Nat. Genet. 50, 1327-1334 (2018).

12. Aguet, F. et al. The GTEx Consortium atlas of genetic regulatory effects across human tissues. BioRxiv (2019) doi:10.1101/787903. 
13. Mohammadi, P., Castel, S. E., Brown, A. A. \& Lappalainen, T. Quantifying the regulatory effect size of cis-acting genetic variation using allelic fold change. Genome Res. 27, 18721884 (2017).

14. Castel, S. E. et al. A vast resource of allelic expression data spanning human tissues. Genome Biol. 21, 234 (2020).

15. Mohammadi, P. et al. Genetic regulatory variation in populations informs transcriptome analysis in rare disease. Science 366, 351-356 (2019).

16. Ferraro, N. M. et al. Transcriptomic signatures across human tissues identify functional rare genetic variation. Science 369, (2020).

17. Kim-Hellmuth, S. et al. Cell type-specific genetic regulation of gene expression across human tissues. Science 369, (2020).

18. Sajuthi, S. P. et al. Type 2 and interferon inflammation regulate SARS-CoV-2 entry factor expression in the airway epithelium. Nat. Commun. 11, 5139 (2020).

19. Brandt, M. et al. An autoimmune disease risk variant: A trans master regulatory effect mediated by IRF1 under immune stimulation? PLoS Genet. 17, e1009684 (2021).

20. Li, X. et al. The impact of rare variation on gene expression across tissues. Nature $\mathbf{5 5 0}$, 239-243 (2017).

21. Lee, Y., Francesca, L., Pique-Regi, R. \& Wen, X. Bayesian Multi-SNP Genetic Association Analysis: Control of FDR and Use of Summary Statistics. BioRxiv (2018) doi:10.1101/316471.

22. Taylor-Weiner, A. et al. Scaling computational genomics to millions of individuals with GPUs. Genome Biol. 20, 228 (2019).

23. NHLBI Trans-Omics for Precision Medicine (TOPMed). https://www.nhlbiwgs.org.

24. Wang, D. et al. Comprehensive functional genomic resource and integrative model for the human brain. Science 362, (2018).

25. Castel, S. E., Mohammadi, P., Chung, W. K., Shen, Y. \& Lappalainen, T. Rare variant 
phasing and haplotypic expression from RNA sequencing with phASER. Nat. Commun. 7, 12817 (2016).

26. Zou, H. \& Hastie, T. Regularization and variable selection via the elastic net. J. Royal Statistical Soc. B 67, 301-320 (2005).

27. Wang, G., Sarkar, A. K., Carbonetto, P. \& Stephens, M. A simple new approach to variable selection in regression, with application to genetic fine-mapping. BioRxiv (2018) doi:10.1101/501114.

28. Gusev, A. et al. Integrative approaches for large-scale transcriptome-wide association studies. Nat. Genet. 48, 245-252 (2016).

29. Keys, K. L. et al. On the cross-population generalizability of gene expression prediction models. PLoS Genet. 16, e1008927 (2020).

30. Shang, L. et al. Genetic Architecture of Gene Expression in European and African Americans: An eQTL Mapping Study in GENOA. Am. J. Hum. Genet. 106, 496-512 (2020).

31. Mogil, L. S. et al. Genetic architecture of gene expression traits across diverse populations. PLoS Genet. 14, e1007586 (2018).

32. Buil, A. et al. Gene-gene and gene-environment interactions detected by transcriptome sequence analysis in twins. Nat. Genet. 47, 88-91 (2015).

33. Petrovski, S. et al. The intolerance of regulatory sequence to genetic variation predicts gene dosage sensitivity. PLoS Genet. 11, e1005492 (2015).

34. Martin, A. R. et al. Clinical use of current polygenic risk scores may exacerbate health disparities. Nat. Genet. 51, 584-591 (2019).

35. Gay, N. R. et al. Impact of admixture and ancestry on eQTL analysis and GWAS colocalization in GTEx. Genome Biol. 21, 233 (2020).

36. van de Geijn, B., McVicker, G., Gilad, Y. \& Pritchard, J. K. WASP: allele-specific software for robust molecular quantitative trait locus discovery. Nat. Methods 12, 1061-1063 (2015).

37. Kumasaka, N., Knights, A. J. \& Gaffney, D. J. Fine-mapping cellular QTLs with RASQUAL 
and ATAC-seq. Nat. Genet. 48, 206-213 (2016).

38. Liang, Y., Aguet, F., Barbeira, A. N., Ardlie, K. \& Im, H. K. A scalable unified framework of total and allele-specific counts for cis-QTL, fine-mapping, and prediction. Nat. Commun. 12, 1424 (2021).

39. Stegle, O., Parts, L., Piipari, M., Winn, J. \& Durbin, R. Using probabilistic estimation of expression residuals (PEER) to obtain increased power and interpretability of gene expression analyses. Nat. Protoc. 7, 500-507 (2012). 